\section{SP4-29 NEIGHBOURHOOD-BASED DIFFERENCES IN PHYSICAL ACTIVITY: A DEPRIVED URBAN NEIGHBOURHOOD PROGRAM APPROACH (FITNESS CENTERS-FCS) FOR REDUCING SOCIAL INEQUALITIES IN CHRONIC DISEASES: PRELIMINARY COMPARISONS}

doi:10.1136/jech.2011.142976p.23

\author{
${ }^{1,2} \mathrm{~W}$ Caiaffa, ${ }^{* 1,2} \mathrm{~A}$ Souza, ${ }^{1,2} \mathrm{~J}$ Goston, ${ }^{1,2} \mathrm{~A}$ Dayrell, ${ }^{1,2} \mathrm{C}$ Xavier, ${ }^{1,2} \mathrm{C}$ Comini, \\ $1,{ }^{2}$ F Proietti. ${ }^{1}$ Federal University of Minas Gerais, Belo Horizonte, Minas Gerais, Brazil; \\ ${ }^{2}$ Observatory for Urban Health, Belo Horizonte, Minas Gerais, Brazil
}

Introduction Intervention in vulnerable groups might produce risk factors modification. This study is the baseline description of a health impact study of the implementation of FCs, a community citywide intervention.

Methods A natural step-wedge design compared areas where the intervention were implemented. FCs are community centers located in vulnerable areas of the city, built for fitness classes, staffed by municipality instructors. Individual indicators came from a multistage (census tracts-CT, households, and residents) survey, stratified by distance street buffers created around the planned point interventions, intersected with the CT, giving probabilistic ranging from 0.1 to 0.012 as the distance increase from the site intervention. Demographics, social determinants, health and lifestyles (including Physical activity-PA: $>150 \mathrm{~min} /$ week), social support for PA (PASS) were collected. PA and co-variables were compared across one area that received earlier intervention with all others surveyed, using multiple Poisson regression models.

Results Of the 4048 adults, $54.1 \%$ were female; mean age 41.2 years; $16.1 \%$ reported PA. Comparing estimates across areas with and without FC, age, female gender, smoking and good self-perception of health were inversely related to PA while PASS, schooling and income were directly associated to PA in areas without FC. For those $17.6 \%$ living in the FC catchment area the only associated factor with PA was report of eating fruits 5 times/week; all other factors were no longer associated with PA.

Conclusion Tailored to address health disparities for chronic diseases, interventions in high risk urban environmental might narrow health inequality for some indicators of physical activity.

\section{SP4-30 GLOBAL NUTRITIONAL BURDEN: A MAJOR METHODOLOGICAL CHALLENGE FOR NUTRITIONAL EPIDEMIOLOGICAL RESEARCH}

doi:10.1136/jech.2011.142976p.24

N Slimani.* International Agency for Research on Cancer, Lyon, France

Since the last $20-30$ years we observe a massive increase in the consumption of industrial foods. This rapid nutritional transition observed first in Western countries is now affecting medium and low income countries and vulnerable groups (eg, children), with an accelerated phenomena. Although the consumption of processed foods (incl. the Western-type diet) is hypothesised to be associated with the rapid increase of chronic diseases worldwide, the lack of specifically designed epidemiological studies is the main reason given by the WCRF for its non-conclusive report. A series of methodological issues related to the traditional dietary methodologies used in epidemiology, and the difficulty to estimate and monitor the consumption of processed foods are possible underlying explanations will be presented.

Results From recent studies on the contribution of (highly) industrially processed foods to the diet of a large population sample of the EPIC study (27 centres, 35-75 years, $\mathrm{N}=\sim 37000$ ), using a unique set of detailed and standardised 24-h dietary recalls (EPIC-soft program) and biomarkers of processed foods (trans fatty acid, acrylamide) will be used to illustrate our talk. In addition, the EPICsoft web-based Methodological Platform-centralised platform at IARC developed to use, maintain and disseminate the standardised and validated EPIC-Soft Methodology for international epidemiological and monitoring surveys-will be used as an example of a longstanding international effort (EPIC, EFCOSUM, EFCOVAL, IDAMES, PANCAKE, EFSA) to improve dietary methodologies and contribute to a better estimation and monitoring of dietary exposure during this major nutritional transition phase and formulate targeted recommendations.

\section{SP4-31 EFFECT OF FIRST AID EDUCATION ON FIRST AID KNOWLEDGE AND SKILLS OF COMMERCIAL DRIVERS IN NIGERIA}

doi:10.1136/jech.2011.142976p.25

A 0 Sangowawa, ${ }^{*}$ M C Asuzu, 00 Kale. University of Ibadan/University College Hospital, Ibadan, Ibadan, Oyo State, Nigeria

Introduction The study was conducted to determine the effect of first aid education on the capacity of commercial drivers in Ibadan, Nigeria to provide first aid for accident victims.

Methods A before-and-after study was conducted among 128 drivers- 62 intervention and 66 controls selected by multi-stage sampling. The drivers' first aid knowledge and skills were assessed at baseline, immediately and 3 months post- intervention. The intervention consisted of a 2-day training on first aid while controls were given health education on HIV/AIDS. First aid knowledge scores were generated giving a maximum aggregate score of 24 for each respondent while minimum and maximum obtainable scores for first aid skills were 0 and 25 respectively.

Results Mean first aid knowledge scores for intervention drivers were $11.8( \pm 2.9), 13.9( \pm 2.7)$ and $14.4( \pm 2.2)$ at baseline, immediate and 3 -month post intervention. Corresponding scores for the control group were $11.6( \pm 3.1), 9.4( \pm 3.7)$ and $11.2( \pm 3.7)$. Mean first aid skill scores for intervention drivers were $4.4( \pm 0.9), 20.2$ $( \pm 2.1)$ and $18.1( \pm 4.2)$. Scores for control drivers were $4.1( \pm 1.1)$, $4.1( \pm 1.2)$ and $5.1( \pm 2.3)$ respectively. Repeated measure analysis of variance showed significant differences in first aid knowledge and skills within the intervention group over the three phases of the study $(p<0.001)$. Scores for controls remained about the same.

Conclusion The first aid education led to significant improvement in first aid knowledge and skills of intervention drivers confirming that drivers can be trained as lay first responders in settings were formal emergency medical services for crash victims are poorly developed.

\section{SP4-32 HEALTH AND SUSTAINABILITY: INTERNATIONAL ECOLOGICAL STUDY OF CARBON DIOXIDE EMISSIONS AND LIFE EXPECTANCY}

doi:10.1136/jech.2011.142976p.26

${ }^{1} \mathrm{E}$ Brunner, ${ }^{*}{ }^{1} \mathrm{~K}$ Maruyama. ${ }^{1}$ University College London, London, UK; ${ }^{2}$ Osaka University, Osaka, Japan

Introduction The nature of the relationship between country-level energy consumption and life expectancy has not been examined systematically.

Methods Ecological study of carbon dioxide $\left(\mathrm{CO}_{2}\right)$ emissions per capita and life expectancy at birth using the maximum available sample of countries

Results Among 155 countries $\mathrm{CO}_{2}$ emissions per capita were in the range 0-30 metric tons per year. Life expectancy at birth rose sharply between 0 and $5 \mathrm{t}$ of emissions from 42 (Afghanistan) to 78 (Costa Rica, Chile). Spearman's rank correlation was 0.78 , 